\title{
White and Gray Matter Abnormalities in Manifest Huntington's Disease: Cross-Sectional and Longitudinal Analysis
}

\author{
Wafaa Sweidan (D), Fen Bao, Navid-Seraji Bozorgzad, Edwin George \\ From the Department of Psychiatry, Wayne State University, Detroit, MI (WS); Department of Neurology, Wayne State University, Detroit, MI (FB, EG); and Department of \\ Neurology, University of Michigan, Ann Arbor, MI (NSB).
}

\begin{abstract}
A B S T R A C T
BACKGROUND AND PURPOSE: Early white matter (WM) changes and cortical atrophy in Huntington's disease (HD) are often evident before disease onset and extend through the brain during manifest stages. The trajectory of these brain abnormalities in symptomatic stages remains relatively unexplored. The aim of this study is to investigate how the pattern of WM and gray matter (GM) alterations progress over time.

METHODS: We investigated alterations in brain WM, cortical thickness, and subcortical structures using diffusion and structural magnetic resonance imaging, in manifest HD patients $(n=13)$ compared to age-matched healthy controls $(n=11)$. Imaging and clinical data for the HD group were collected at follow-up (7 months) to explore possible longitudinal changes.

RESULTS: Cross-sectional analyses identified significant posterior cortical thinning $(P<.05)$ and symmetric fractional anisotropy $(F A)$ reduction $(P<.01)$ in brain WM of HD group compared to HC. These changes were strongly correlated with impairment in motor symptoms and processing speed. Subcortical atrophy was significant in caudate, putamen, globus pallidus, and thalamus $(P<.001)$. Regions of interest analysis revealed a significant reduction in FA of the corpus callosum (CC) $(-2.19 \%$, $P<.05)$ upon follow-up, whereas no significant cortical thinning and subcortical atrophy was found.

CONCLUSIONS: This study showed broad GM and WM abnormalities in manifest HD patients. Reductions in FA and cortical thinning correlated significantly with the disturbances of motor and cognitive processing that describe HD. Follow-up assessment showed that the CC is compromised in the absence of detectable GM changes or motor decline, suggesting it plays an important role in disease progression.
\end{abstract}

Keywords: Huntington's disease, white matter, cortical thinning, clinical markers.

Acceptance: Received November 6, 2019, and in revised form February 6, 2020. Accepted for publication February $18,2020$.

Correspondence: Address correspondence to Wafaa Sweidan, University Health Center, Detroit, MI. E-mail: et2975@ wayne.edu

This study has been funded by Biogen MA Inc.

J Neuroimaging 2020;30:351-358.

DOI: $10.1111 /$ jon.12699

\section{Introduction}

Huntington's disease (HD) is an autosomal dominant and progressive neurodegenerative disorder caused by an expansion of the CAG (glutamine) triplet in exon-1 of the huntingtin gene (HTT). ${ }^{1}$ The hallmark of HD neuropathology is the massive loss of medium spiny neurons in the striatum ${ }^{2}$ and with disease progression there is involvement of the cerebral cortex and other subcortical structures. ${ }^{3}$ Clinically, HD is characterized by motor abnormalities, variable mood disturbances, ${ }^{6}$ such as depression, apathy, and irritability, as well as cognitive deterioration ${ }^{4,5}$ cul- $^{-}$ minating in dementia. As symptomology extends beyond the motor dysfunction to involve mood and cognition, prominent cortical and subcortical pathological changes are known to contribute to the clinical status in HD. Morphological magnetic resonance imaging (MRI) studies have demonstrated cortical thinning in primary cerebral areas (sensory, motor, and visual) and higher cortical regions with disease progression, with a relative conservation of the most anterior frontal and inferior temporal regions. ${ }^{3,4}$ However, little is known about the effects of HD on white matter (WM) regions as the disease progresses. The TRACK-ON HD study, a longitudinal study of 72 HD patients over 24 months, demonstrated a hierarchy of WM vulnerability in premanifest $\mathrm{HD}$, where cortico-striatal connections are most affected, followed by interhemispheric and intrahemispheric connections. ${ }^{6}$

Given that changes in structure may manifest as changes in local diffusion of water, diffusion tensor imaging (DTI) can be used to infer WM microstructural changes related to $\mathrm{HD}$ pathology. DTI measures the overall orientation of WM bundles, with a principal outcome measure of fractional anisotropy (FA), which describes the directionality of diffusion and is related to the presence and coherence of orientated structures. ${ }^{7}$ Mean diffusivity (MD) is another DTI parameter that characterizes the overall diffusion and is related to the overall presence of obstacles to diffusion. In this regard, few longitudinal studies are available to characterize the trajectory of gray matter (GM) and WM abnormalities over time and its relation with the clinical status of the patient. The IMAGE-HD study provided the first evidence for a longitudinal reduction in FA in the corpus callosum (CC) and cingulum of the symptomatic HD group over a period of 18 months. ${ }^{8}$ An important question is whether these pathological changes are also detectable at shorter follow-up periods.

In this study, we aim to investigate brain changes associated with HD progression by analyzing microstructural changes in WM regions, cortical thickness, and subcortical atrophy over a 
period of 7 months, in an attempt to identify key biomarkers for disease progression. This will allow us to identify short-term brain changes in the absence of significant clinical deterioration. For this purpose, initially we performed tract-based spatial statistics (TBSS) to investigate abnormalities in WM and study its correlation with CAG-repeats length and clinical measures of motor severity and cognition dysfunction. Second, we aimed to investigate the longitudinal changes in FA and MD upon follow-up. In addition, we studied the longitudinal changes of the well-documented cortical abnormalities in manifest HD patients. We predicted that upon follow-up, cortical thinning will extend to more areas compared to baseline, preceding the cognitive deterioration that would be expected. We also expected that FA will be significantly lower upon follow-up.

\section{Methods}

All aspects of this study, including subject recruitment and consent process, were approved by the Institutional Review Board at Wayne State University and Detroit Medical Center.

\section{Participants}

Thirteen genetically tested HD individuals (CAG repeats $\geq 40$ ) were recruited from the Wayne State University/Detroit Medical Center Movement Disorder Clinic. Eleven age-matched healthy controls $(\mathrm{HC})$ without a family history of neurologic diseases were also enrolled from the pool of family members and care givers who visited the clinic. All HD participants underwent brain MRI, neuropsychological assessment, and Unified Huntington's Disease Rating Scale ${ }^{9}$ scoring by a movement disorder neurologist (EG). All HD participants were diagnosed as symptomatic based on neurologic evaluation and UHDRS motor score $>5 .^{10}$ Global cognition was evaluated using the Montreal Cognitive Assessment (MoCA) ${ }^{11}$; information processing speed and working memory were evaluated using Symbol Digit Modalities Test-oral version (SDMT). Raw SDMT scores were converted to $z$-scores based on published age and gender-based normative data. ${ }^{12}$ Impaired SDMT performance was defined as $z$-score $\leq-1.5$. HD participants returned for their follow-up scan 7 months later.

All imaging sequences were acquired on a 3-Tesla Verio Siemens scanner with a 32-channel head coil and included: T1-weighted MPRAGE sequence (176 slices; slice thickness of $1.3 \mathrm{~mm} ; .7 \mathrm{~mm} \times .7 \mathrm{~mm}$ in-plane resolution; Repetition time (TR)/Echo Time (TE)/Inversion time $(\mathrm{TI})=1,680 / 3.52 / 900 \mathrm{~ms}$; flip angle $=9^{\circ}$ ) and diffusionweighted (DW) axial pulsed-gradient spin-echo EPI (TR/TE = $10,400 / 126 \mathrm{~ms}$; flip angle $=90^{\circ} ; 46$ contiguous slices; $1.28 \mathrm{~mm}$ $\times 1.28 \mathrm{~mm} \times 3 \mathrm{~mm}$; acquisition matrix $128 \times 128$ with $b$-value $=1,000 \mathrm{~s} / \mathrm{mm}^{2}[20 \mathrm{scans}]$ and $b$-value $=0 \mathrm{~s} / \mathrm{mm}^{2}$ [two scans]). T2 FLAIR images $(1 \mathrm{~mm} \times 1 \mathrm{~mm} \times 3 \mathrm{~mm}$; flip angle $=$ $150^{\circ} ; \mathrm{TR} / \mathrm{TE} / \mathrm{TI}=9,000 / 128 / 2500 \mathrm{~ms}$ ) were acquired to assess any pathological lesions, including microvascular angiopathy, prior stroke, or other lesions.

\section{MRI Preprocessing}

DW Images were preprocessed using the FSL software (Version 5.0.4; http://www.fmrib.ox.ac.uk/fsl/). All DTI images were corrected for eddy current-induced distortions and head motion using the FSL tool eddy_correct and the gradient directions (bvecs) were rotated accordingly using fdt_rotate_bvecs
(Leemans and Jones, 2009). Diffusion data of $1 \mathrm{HD}$ subject at baseline and $1 \mathrm{HD}$ subject at follow-up showed severe motion artefacts and were excluded from analysis.

Maps of FA and MD were created by fitting the diffusion tensor images with weighted least-squares using "dtifit" tool from the FSL toolbox to create.

\section{TBSS Analysis}

TBSS was performed using FSL as described previously. ${ }^{13}$ In the baseline analysis, FA maps of the HD and HC participants were used to generate a group-wise skeleton of WM tracts, thresholding the FA skeleton at .2 and resulting in a mask of 128,432 pixels. Between-group (HD vs. HC) $t$-tests were performed using the function "Randomise" from $\mathrm{FSL}^{14}$ on the skeletonized WM fiber tracts for FA and MD maps. The number of Randomise permutations was 10,000 with threshold-free cluster enhancement (TFCE) and corrected for multiple comparisons using family wise error correction.

To explore the trend of FA changes in time, a subject-specific template was created to enhance individual subject image alignment across time points. ${ }^{15}$ Baseline FA map was registered to follow-up FA map and vice versa (follow-up to baseline). The halfway point between the two registered images was computed. Then, a halfway registration was performed between the baseline FA and follow-up FA map for each subject. Next, a subject-specific template was created by averaging the two halfway registered FA images. The subject-specific template was transformed to MNI (Montreal Neurological Institute) space. The resulting transform was then applied to the baseline and follow-up FA maps in halfway space. After registration of the halfway images to MNI space, TBSS processing routines was continued as above.

Between time points (baseline and follow-up) comparisons of HD group were then analyzed using paired $t$-test (TBSS, TFCE enabled, 5,000 permutation) for FA and MD measures, which enabled us to investigate the longitudinal changes in diffusion metrics in the HD group.

Finally, we investigated voxelwise correlations of FA with clinical variables in the HD group using TBSS. All resulting statistical maps were thresholded at a family-wise error corrected $P$-value $(\mathrm{pFWE})<.05$. For clusters showing significant correlation of FA with clinical variables, we extracted mean FA values of all HD subjects from the tracts of interest and plotted them against UHDRS and SDMT scores to test their relationship using Pearson's correlation analysis. All the general linear model (GLM) designs included demeaned age and sex as nuisance covariates. All the results were anatomically localized using the JHU ICBM-DTI-81 White-Matter Labels and the JHU White-Matter Tractography atlases.

\section{FreeSurfer Analysis Pipeline}

FreeSurfer (version 5.3.0; http://surfer.nmr.mgh.harvard.edu) was used to derive measures of cortical thickness and deep GM nuclei volume. The automated procedures for cortical reconstruction and volumetric segmentation have been previously described. ${ }^{16}$ Subcortical GM nuclei volumes included in the analysis were as follows: caudate, putamen, globus pallidum, and thalamus. For each hemisphere, a GLM was computed vertex-by-vertex for analysis of cortical thickness, accounting for the effects of gender and age. 
The design matrix was created using DODS (different offsets, different slopes) that assumes different offsets but a different impact of duration between groups (different slope), which might be plausible in this study. Cortical maps were smoothed using a 10-mm full width at half maximum Gaussian kernel and the results were visualized by overlaying significant cortical areas onto semi-inflated cortical surfaces. Multiple comparisons were corrected with a false discovery rate $($ FDR $)<.05$.

The association between cortical morphometric measures and clinical measures in each clinical group was also examined using Query, Design, Estimate, Contrast application (QDEC) in FreeSurfer. CAG repeats, measures of motor severity as measured by UHDRS-III, global cognitive function, and processing speed as measured by the MOCA and SDMT were included in the analyses individually as continuous covariates $(\mathrm{FDR}<.05)$. Finally, FreeSurfer's paired analysis pipeline was used to investigate cortical thickness changes in HD group at baseline versus follow-up.

\section{Statistics}

Demographic and cognitive data were analyzed in R-Studio (RStudio Team, 2016, version 3.4.4), and included $\chi^{2}$ for categorical and Wilcoxon ranks sum test for continuous variables. Assumptions for normality were tested using the Shapiro-Wilk normality test.

Average subcortical volumes between right and left hemispheres, were compared between groups first using MANCOVA, with age, sex, and intracranial volume (ICV) as covariates. After obtaining significant group differences, univariate differences were then analyzed using a GLM with age, gender, and ICV as covariates.

Linear mixed effects models were used to investigate the trajectory subcortical volume with time in the HD group. For each subcortical volume (average of right and left hemispheres), we modeled their interaction with time treated as "fixed effect," while statistically controlling for gender, age at baseline, and the effects of randomly selected participants ("random effects"). $P$-value of .05 was used as threshold for significance in all of statistical analyses.

\section{Results}

\section{Demographics and Group Characteristics}

Table 1 summarizes the demographic and disease characteristics of the HD and control groups. Age and sex did not differ significantly between groups. Due to a small sample size, there was a slight mismatch in the distribution of genders between the control group and the HD group. At follow-up, only 11 HD patients underwent brain MRI on the same scanner, cognitive assessment, and UHDRS scoring by the same neurologist (EG), 1 patient was not able to do the MRI, and 1 withdrew from the study. Global cognition as assessed by MoCA was less than 26 in $77 \%$ of the patients, indicating an overall cognitive impairment.

Performance on individual subscores of the MoCA was analyzed, with impairment defined as less than $60 \%$ of the maximum score (Table 2). The most impaired items in the MoCA were the language $(1.2 \pm 1.2 ; 41 \%)$ and delayed recall $(2.8 \pm 1.6$; $57 \%)$. This indicates that the language and delayed recall subsets contributed the most to poor scores on the MoCA.
Table 1. Demographic Data of Huntington Patients and Healthy Controls

\begin{tabular}{|c|c|c|c|}
\hline Group & Huntington & Controls & $P$-value \\
\hline $\begin{array}{c}\text { Number of } \\
\text { subjects }\end{array}$ & 13 & 11 & - \\
\hline Age, mean $\pm \mathrm{SD}$ & $57.69 \pm 12.39$ & $57 \pm 15$ & $\begin{array}{l}.5 \text { (Wilcoxon } \\
\text { rank) }\end{array}$ \\
\hline Gender (F:M) & $(7: 6)$ & $(9: 2)$ & $\begin{array}{l}.2 \\
\text { (Chi-square) }\end{array}$ \\
\hline $\begin{array}{l}\text { CAG repeats, } \\
\text { mean } \pm \mathrm{SD} \\
\text { (range) }\end{array}$ & $\begin{array}{l}43.2 \pm 2.3 \\
(40-47)\end{array}$ & N/A & - \\
\hline $\begin{array}{l}\text { Follow-up, mean } \\
\quad \pm \mathrm{SD}\end{array}$ & $7.1 \pm 1.0$ months & N/A & - \\
\hline UHDRS & & N/A & .268 (paired \\
\hline Baseline $(n=13)$ & $45.78 \pm 20.9$ & & $t$-test) \\
\hline $\begin{array}{l}\text { Follow-up } \\
\quad(n=12)\end{array}$ & $40.5 \pm 19.17$ & & \\
\hline $\begin{array}{l}\text { Percent change } \\
\quad(n=12)\end{array}$ & $-4.89 \pm 1.47 \%$ & & \\
\hline $\mathrm{MoCA}$ & & NA & .943 (paired \\
\hline Baseline $(n=13)$ & $20.3 \pm 5.63$ & & $t$-test) \\
\hline $\begin{array}{l}\text { Follow-up } \\
\quad(n=12)\end{array}$ & $20.8 \pm 6.67$ & & \\
\hline $\begin{array}{l}\text { Percent change } \\
\quad(n=12)\end{array}$ & $.4 \pm .06 \%$ & & \\
\hline SDMT $z$-score & & N/A & .05089 \\
\hline Baseline $(n=13)$ & $-3.0 \pm 2.02$ & & (paired $t$-test) \\
\hline $\begin{array}{l}\text { Follow-up } \\
\quad(n=12)\end{array}$ & $-3.2 \pm 2.32$ & & \\
\hline $\begin{array}{l}\text { Percent change } \\
\quad(n=12)\end{array}$ & $-8.6 \pm .18 \%$ & & \\
\hline
\end{tabular}

$\mathrm{SD}=$ standard deviation $; \mathrm{F}=$ Female $; \mathrm{M}=$ Male $\mathrm{CAG}=$ cytosine-adenineguanine; $\mathrm{N} / \mathrm{A}=$ not applicable; $n=$ number of subjects.

Table 2. Performance on Individual Subtests of MoCA in Huntington Group

\begin{tabular}{lcccc}
\hline & \multicolumn{4}{c}{$\begin{array}{c}\text { Average } \\
\text { Mean \% } \\
\text { Score }\end{array}$} \\
$\begin{array}{l}\text { MocA subtest } \\
\text { percent change } \\
\text { upon follow-up } \\
(\boldsymbol{n}=\mathbf{1 2})\end{array}$ & $\begin{array}{c}\boldsymbol{P} \text {-value } \\
\text { (paired } \\
\text { t-test) }\end{array}$ \\
\hline Max score) & $n=12 \mathrm{HD}$ & & & \\
Visuoexecutive (5) & $3 \pm 1.8$ & 60 & $-2.63 \%$ & .8 \\
Naming (3) & $2.6 \pm .7$ & 87 & $0 \%$ & 1 \\
Attention (6) & $3.8 \pm 1.53$ & 63 & $0 \%$ & 1 \\
Language (3) & $1.2 \pm 1.2$ & $41^{*}$ & $6.25 \%$ & .75 \\
Abstraction (2) & $1.3 \pm .8$ & 65 & $12.5 \%$ & .5 \\
Delayed recall (5) & $2.8 \pm 1.6$ & $57^{*}$ & $9.09 \%$ & .66 \\
Orientation (6) & $5.5 \pm 1.1$ & 91 & $-4.54 \%$ & .34 \\
\hline
\end{tabular}

$\mathrm{SD}=$ standard deviation; $n=$ number of subjects.

${ }^{*}$ Less than $60 \%$ of the maximum score.

A more specific cognitive domain, processing speed as assessed by SDMT, revealed more severe cognitive deterioration, where average $z$ score in HD group was $-3.0 \pm 2.0$. Upon follow-up, there were no significant changes in UHDRS and MoCA scores in HD group. However, SDMT scores decreased significantly, from -3.0 at baseline to $-3.2 \mathrm{SD}$ below the mean $(P=.05)$ at follow-up.

\section{HD-Related Effects on WM}

TBSS analysis showed symmetrical lower FA and higher MD in HD group compared to HC (see Fig 1). Area of significant WM FA reduction included the cingulum, genu, body, and splenium 


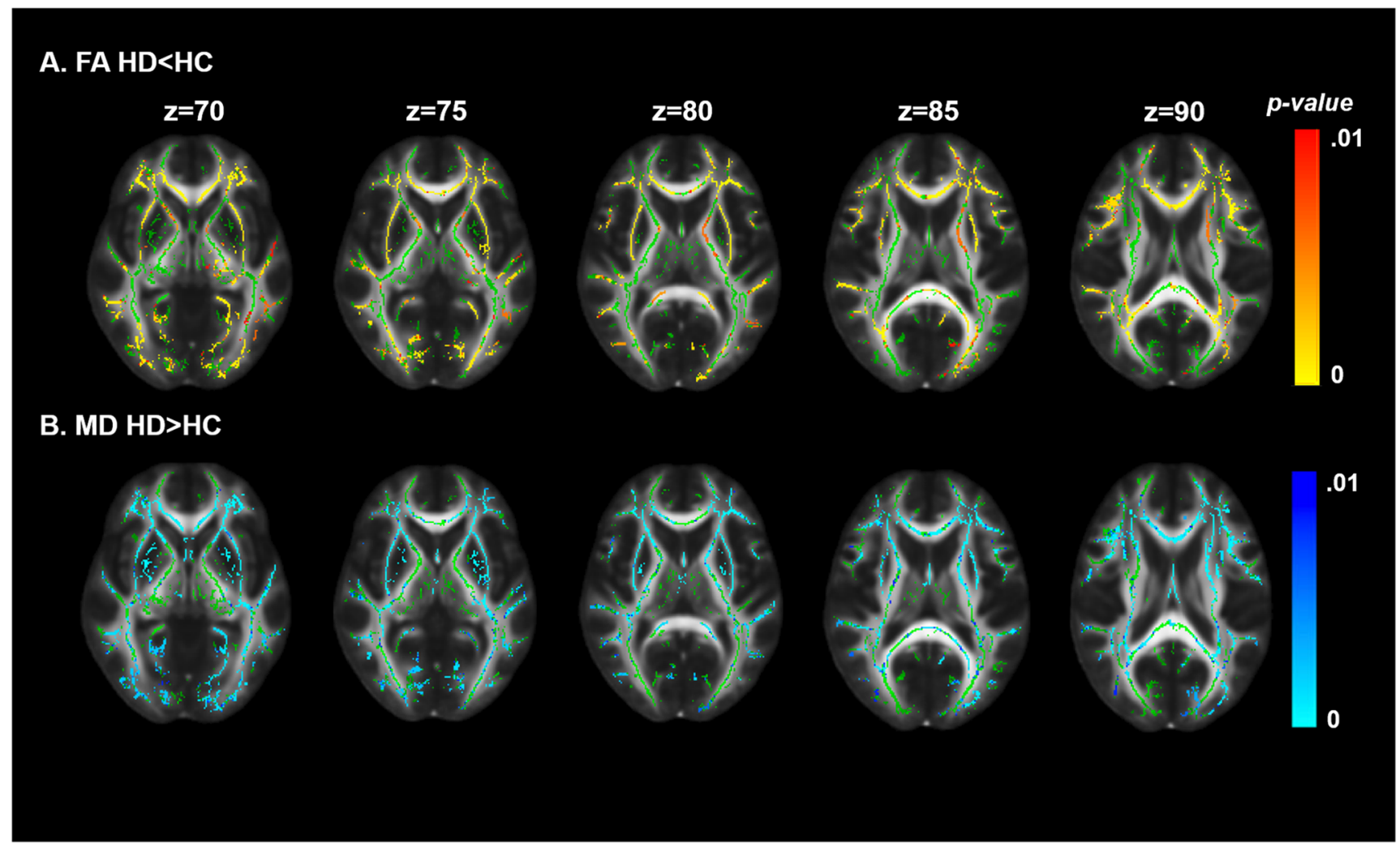

Fig 1. White matter abnormalities in Huntington disease patients compared to healthy controls. The FA skeleton is represented in green (panels $A$ and B), and significant reductions in FA values shown in yellow-red (panel A). Mean diffusivity results (panel B) show areas of significantly higher mean diffusivity in HD group (light blue-dark blue colors) overlaid with FA skeleton (green). Threshold-free cluster enhancement $P<.01$. Group differences are overlaid on the FA template (FMRIB58 $1 \mathrm{~mm}$ ).

of CC, external capsule, anterior and posterior limb of internal capsule, posterior thalamic radiation, superior corona radiata, and superior longitudinal fasciculus $(10,000$ permutations, TFCE corrected, $P<.01)$. Similarly, areas of higher MD in HD group compared with HC were widespread and included, beside areas of lower FA, the anterior corona radiate and fornix.

Voxelwise correlations between DTI parameters and clinical variables in HD group revealed several regions with correlations to specific clinical variables. FA was negatively correlated with UHDRS score (severity of motor symptoms) in genu, body, and splenium of $\mathrm{CC}$ and in posterior thalamic radiation (Fig 2A). In addition, FA was positively correlated with SDMT $z$-score in more posterior regions (Fig 2B). Lower SDMT scores correlated with FA in the posterior corona radiata, superior longitudinal fasciculus, posterior thalamic radiation, body and splenium of CC, sagittal stratum, and the retrolenticular part of internal capsule. Furthermore, we extracted FA values from some of the WM areas that showed correlation with clinical measures, such as the $\mathrm{CC}$ subregions, thalamic radiation, and internal capsule. We found a significant negative correlation between FA and UHDRS in the genu and thalamic radiation, and a positive correlation between FA and SDMT $z$-scores in the internal capsule and thalamic radiation as well (Fig 3). There were no clusters of significant correlation between FA values and MoCA score or number of CAG repeats.

Longitudinal TBSS analysis of FA and MD in the HD group revealed no significant diffusivity changes for the follow-up period of 7 months. Region of interest analysis of the three CC sub-regions-anterior, body, and posterior-revealed significant decreased in FA upon follow-up in the HD group in the anterior (baseline: $.435 \pm .021$ vs. follow-up: .426 $\pm .022, P=.0274$ ) and posterior (baseline: $.575 \pm .028$ vs. follow-up: $.562 \pm .030$, $P=.0421) \mathrm{CC}$, where the average percentage FA change in both regions was $2.19 \pm .13 \%$ (Fig 4 ). The reduction in FA did not correlate with the decreased performance on the SDMT task, which might indicate another mechanism mediating this decline.

\section{Cortical Thinning and Subcortical Atrophy in HD}

Group comparisons of cortical parcellation maps showed significantly lower cortical thickness in the primary the precentral (primary motor) and left postcentral (primary sensory) gyri. Moreover, significant cortical thinning was present in association cortical areas: the right lingual and fusiform gyri, and left supramarginal area in $\mathrm{HD}$ compared to $\mathrm{HC}$ (Fig 5). In addition, cortical thinning extended to right superior temporal and right and left inferior parietal lobules.

Cortical thinning in the HD group correlated negatively with higher motor scores on the UHDRS in the postcentral gyrus, superior frontal lobe, superior and middle temporal lobe, inferior parietal lobe, pars opercularis and pars triangularis, and lingual and lateral orbitofrontal gyrus, whereas lower MoCA scores correlated positively with cortical thinning in the lingual and fusiform gyri, supramarginal gyrus, and lateral orbitofrontal lobe. Similarly, lower $z$-scores on SDMT were associated with cortical thinning in the medial orbitofrontal lobe, middle and inferior temporal lobe, lingual and supramarginal gyri, superior frontal, superior parietal, and lateral occipital lobes 


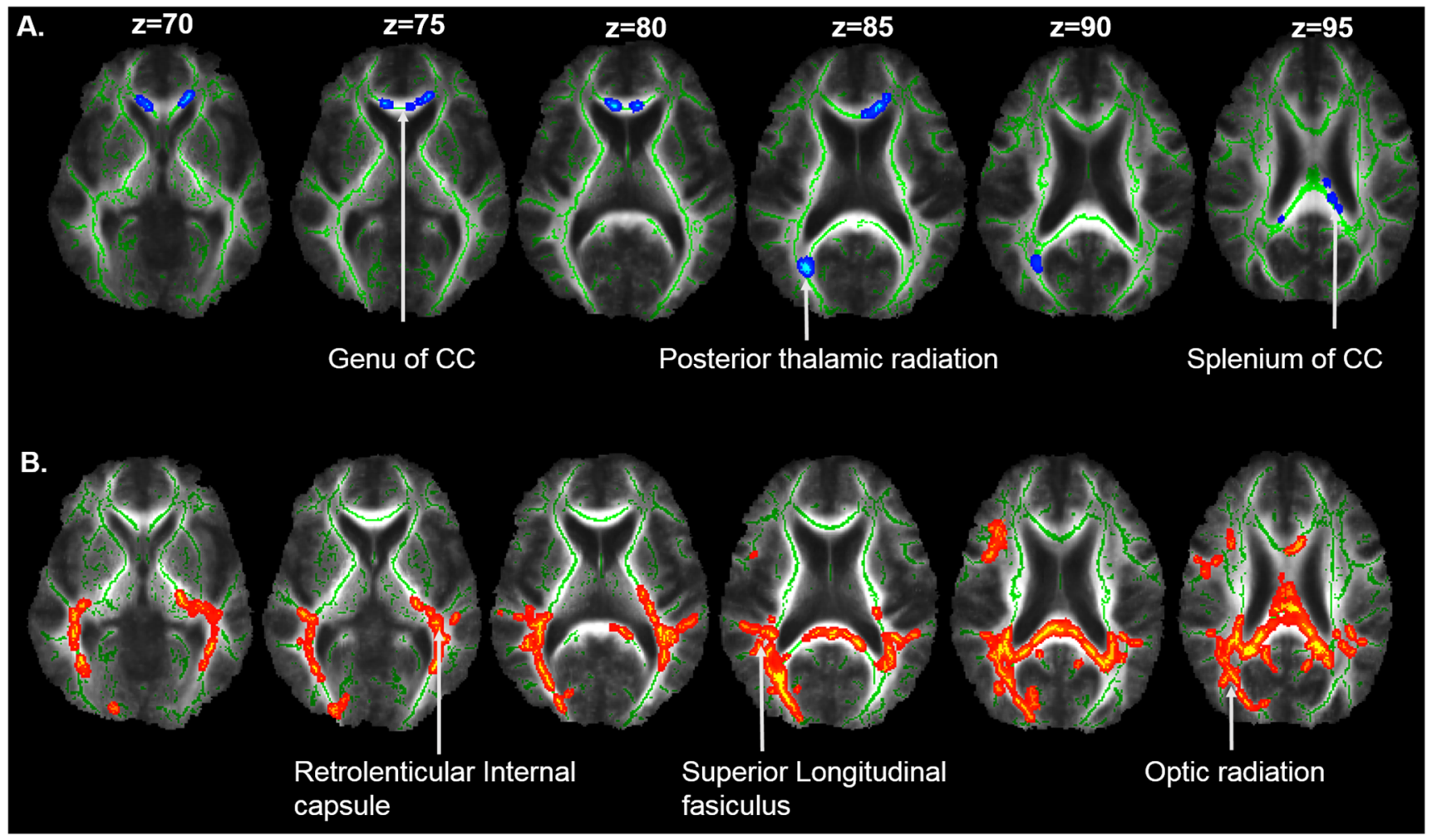

Fig 2. Results of correlation in HD patients between FA and UHDRS. (A) Blue voxels show white matter clusters where the FA is negatively correlated with UHDRS score and FA in genu and splenium of corpus callosum (CC) and optic radiation. (B) White matter clusters showing a significant positive correlation (represented in red) between SDMT score and FA in posterior corona radiation, superior longitudinal fasciculus, inferior longitudinal fasciculus, internal capsule, and optic radiation. Results are overlaid on the mean FA skeleton (green). $P<.05$ corrected for multiple comparisons using threshold-free cluster enhancement.

(Fig 6). Paired analysis in FreeSurfer revealed no significant increase in cortical thinning for the follow-up period in the HD group.

MANCOVA analysis between the HD and HC group with the right and left average volume of caudate, putamen, globus pallidus and thalamus, as dependent variables, showed a significant overall group effect (Wilks Type III F $=.15355, P=9.924$ $\times 10^{-7}$ ). GLM analysis controlling for age, sex, and ICV demonstrated significant group differences in caudate (mean $\pm \mathrm{SEM}$, $3.2 \pm .1 \mathrm{~mL}$ in $\mathrm{HC}$ vs. $\left.2.1 \pm .2 \mathrm{~mL} ; P=2.548 \times 10^{-5}\right)$, putamen $\left(5.0 \pm .3 \mathrm{~mL}\right.$ in $\mathrm{HC}$ vs. $\left.2.7 \pm .2 \mathrm{~mL} ; P=5 \times 10^{-7}\right)$, pallidum $\left(1.5 \pm .05 \mathrm{~mL}\right.$ in $\mathrm{HC}$ vs. $.9 \pm .05 \mathrm{~mL}$ in $\left.\mathrm{HD} ; P=1.508 \times 10^{-7}\right)$, and thalamus $(6.7 \pm .3 \mathrm{~mL}$ in $\mathrm{HC}$ vs. $6.1 \pm .3 \mathrm{~mL}$ in $\mathrm{HD} ; P=$ $\left.3.180 \times 10^{-3}\right)$, corrected for multiple comparisons. Follow-up analysis did not reveal any significant changes in subcortical volume at follow-up versus baseline.

\section{Discussion}

The present study sought to identify short-term variations in major WM areas, as well as cortical and subcortical atrophy in $\mathrm{HD}$ subjects compared to $\mathrm{HC}$, and to what degree these brain changes are associated with severity of the motor and cognitive dysfunction. A number of findings emerged from the results: (1) FA in HD group was correlated with motor and cognitive impairments and continued to decrease upon follow-up in the CC; (2) HD subjects showed significant cortical thinning at baseline compared to HC participants, which did not change upon follow-up; (3) processing speed was significantly worse upon follow-up in HD group.
Findings from previous studies have suggested that HD has complex effects on WM microstructure. The earliest WM changes are seen in the premanifest stage around the striatum, in the CC, and in posterior WM tracts, ${ }^{17-18}$ manifested as reduced FA and increased $\mathrm{MD}$, and this insult to deep WM extends to further tracts as the disease progresses into the manifest stages. Although decreases in FA may arise due to multiple pathological changes including de-myelination or axonal damage, the exact biological process underlying these changes remains under investigation. Recent evidence suggests that myelin breakdown contributes to WM impairments in pathways of the basal ganglia and motor systems, evident by reductions in the macromolecular proton fraction from quantitative magnetization transfer imaging. ${ }^{19}$ Our current findings on microstructural WM alterations are in line with previous work.

We observed symmetrically reduced FA combined with higher MD in WM of manifest HD subjects covering essentially the whole brain. TBSS showed symmetrically lower FA in the WM of commissural fibers (genu, body, and splenium of $\mathrm{CC}$ ), projection fibers (external capsule, thalamic radiation, and corona radiata), association fibers (superior longitudinal fasciculus), and cingulum and sagittal stratum.

Damage to the $\mathrm{CC}$ can interfere with its role in relaying sensory, motor, and cognitive information between cortical regions. In the HD group, we found a significant negative correlation between FA and the motor severity score in the genu and splenium of the CC. In addition, FA in the splenium, superior longitudinal fasciculus (SLF), optic radiation, and 
A

$$
r=-.7, \quad p=.009
$$

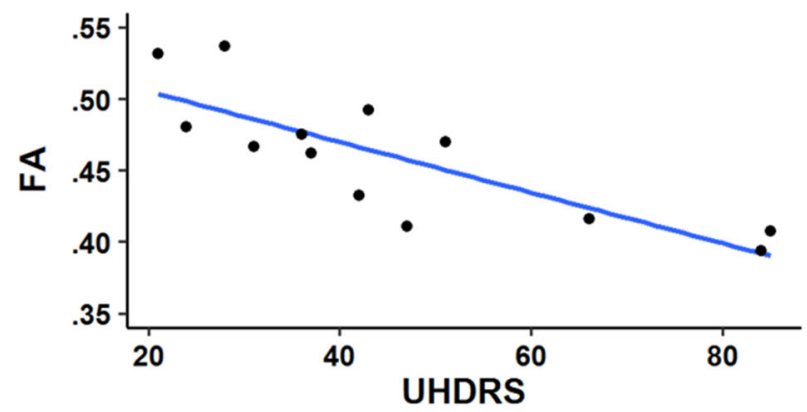

C

$$
r=.6, p=.03
$$

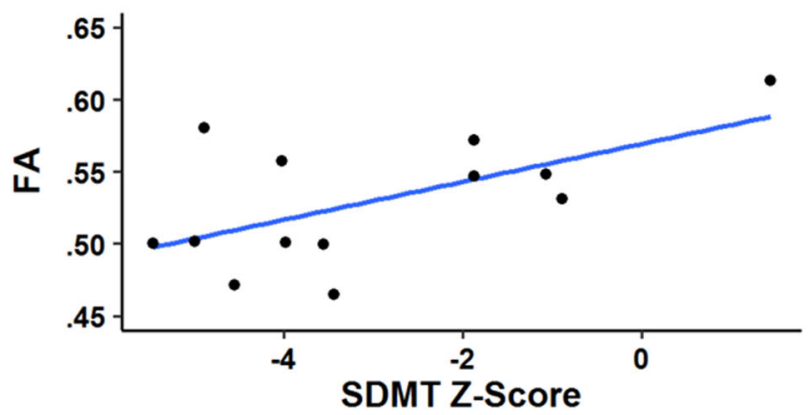

B

$r=-.9, p=.003$

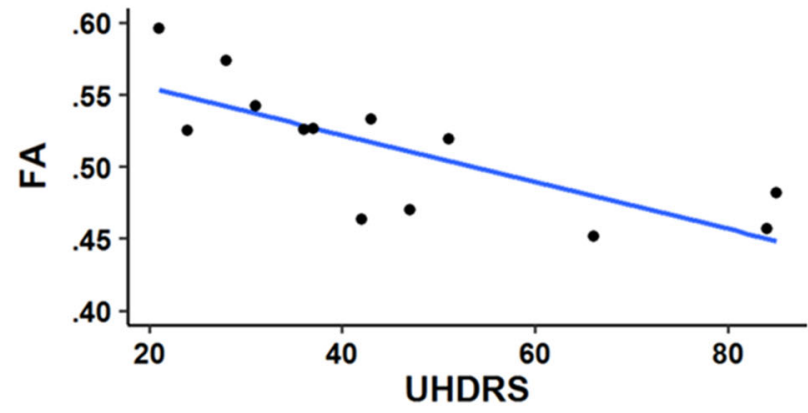

D

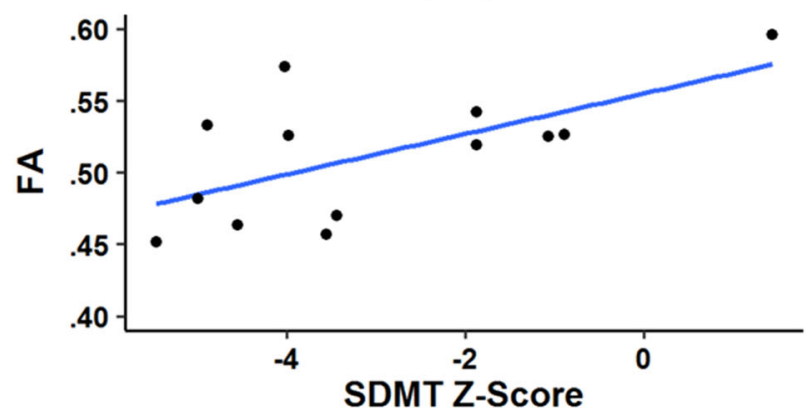

Fig 3. Correlation scatterplot showing relationship between FA and baseline clinical status. UHDRS score correlated negatively with FA in genu (A) of corpus callosum and posterior thalamic radiation (B). SDMT score correlated positively with FA left retrolenticular internal capsule (C) and posterior thalamic radiation (D). The $r$-values represent Pearson's correlation coefficient.

\section{Structure Anterior Corpus Callosum Posterior Corpus Callosum}

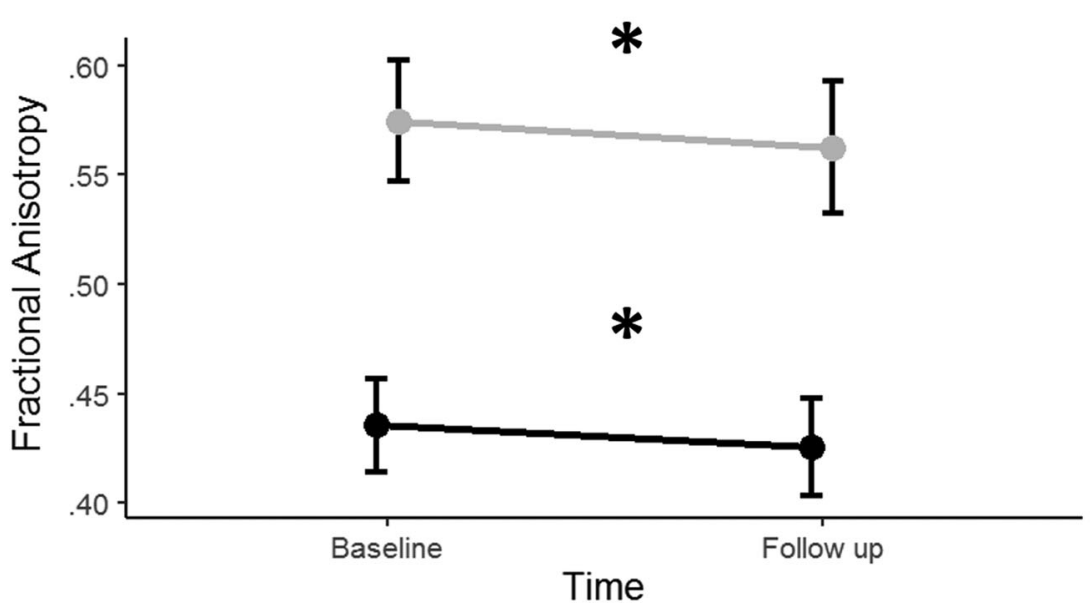

Fig 4. Longitudinal decrease in FA of corpus callosum (CC) in HD group. Regional longitudinal analysis revealed significant decrease in FA in the anterior and posterior portions of the CC after $7 \pm 1.0$ months from baseline scan. Data are represented as mean $\pm \mathrm{SEM}$. ${ }^{*} P<.05$ for comparison of FA at baseline versus follow-up.

internal capsule correlated positively with performance on the SDMT, a measure of processing speed (Fig 2). The involvement of the splenium and optic radiation could be explained by the need to transfer information between hemispheres during the task and the visuospatial demands. ${ }^{20}$ The SLF is also a prominent contributor to processing speed through frontoparietal integration. ${ }^{21}$ These selective WM changes highlight the degenerative effects of HD pathology on specific
WM pathways, which contributes to motor impairment and reduced processing speed.

For the regional longitudinal analysis, we chose to focus on the $\mathrm{CC}$ and cingulum. This was based on findings from the IMAGE-HD study that showed a reduction in FA over 18 months in both the CC and middle cingulum WM, in symptomatic HD subjects compared to HC. ${ }^{8}$ Thus, we hypothesized that our $\mathrm{HD}$ cohort will also show a reduction in FA in the $\mathrm{CC}$ 


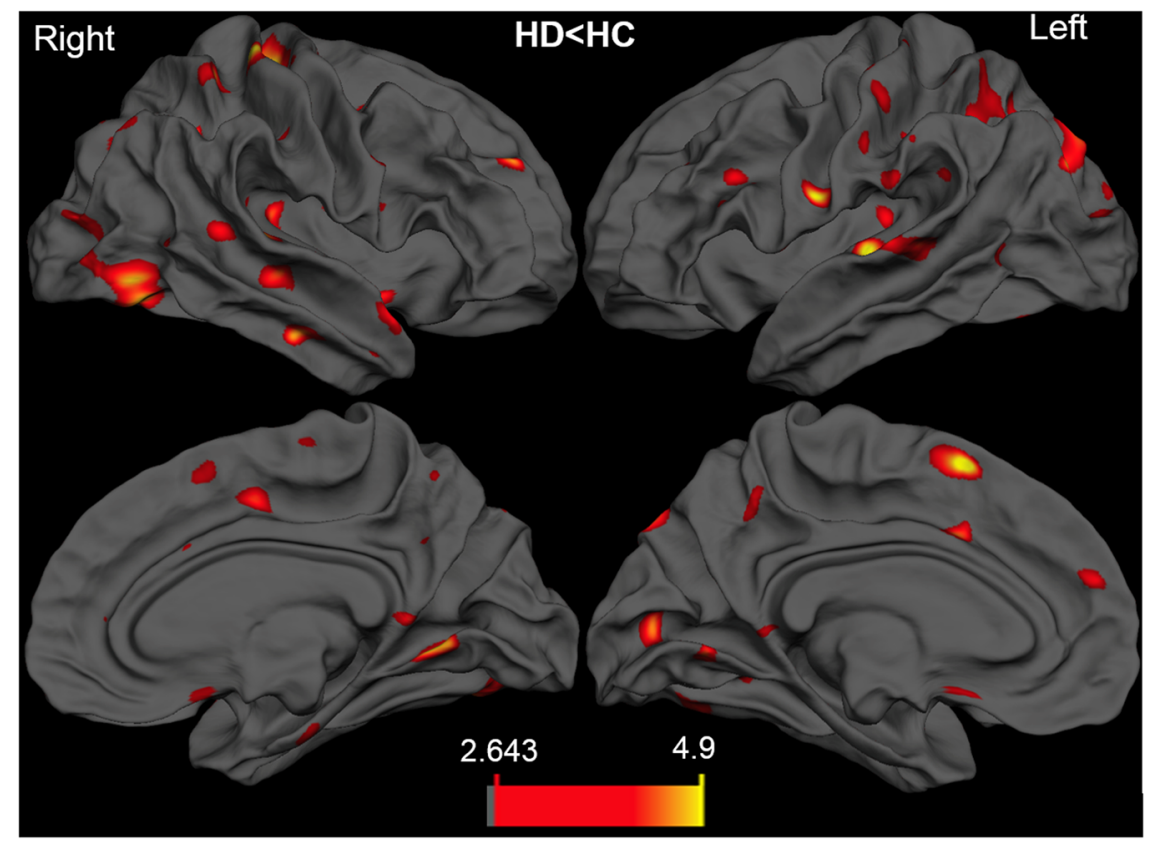

Fig 5. Vertex-wise comparisons of cortical thickness between controls and HD patients. Cortical thinning in HD patients was apparent (yellowred). The color scale bar shows the logarithmic scale of $P$ values $(-\log 10)$. All results were adjusted for multiple comparisons (FDR $P<.05)$ and corrected for age and sex.

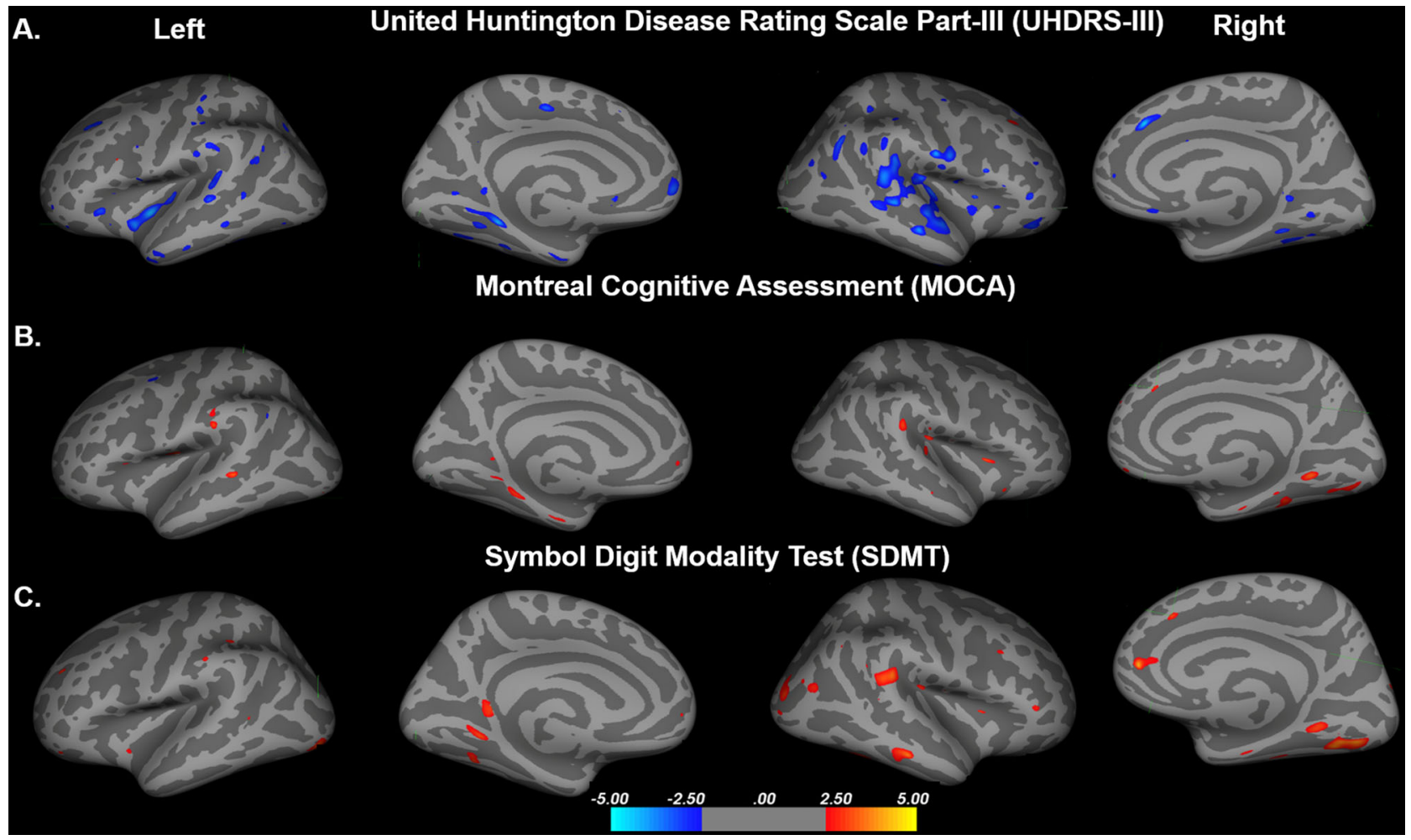

Fig 6. Associations between cortical thinning and motor and cognitive deficits in patients with HD. Significant vertex-wise correlation between cortical thinning and (A) UHDRS-III motor scores; (B) MoCA (C) and SDMT scores. The color scale bar shows the logarithmic scale of $P$ values $(-\log 10)$. All results were adjusted for multiple comparisons (FDR $P<.05)$ and corrected for age and sex.

and middle cingulum matter. Our region of interest analysis for the three $\mathrm{CC}$ sub regions and the cingulum revealed significant reduction of FA in the anterior and posterior regions of the $\mathrm{CC}$. These results suggest that microstructural abnormalities in this WM region continue during disease manifestation stages, de- spite negative results in whole brain longitudinal TBSS analysis. The small sample size can explain why we were not able to detect FA changes at the whole brain level. Hence, increasing our sample size in the future will be necessary to better characterize longitudinal changes in WM over time. 
The distribution of GM atrophy in our HD subjects involved the primary motor and sensory areas and parietal and occipital regions, in line with prior reports showing the earliest cortical atrophy in posterior cortical regions, which later extends to involve midline and frontal regions in manifest stage. ${ }^{22}$ Cortical thinning potentially contributes to the disruption of both motor and cognitive processing that characterize HD. Indeed, a strong positive association was found between performance on the processing speed task and atrophy in the lingual, fusiform, cuneus, lateral occipital, and inferior parietal lobes. Similarly, a strong negative association between UHDRS-motor score and cortical thinning was recorded in more regions including both posterior and anterior areas. The absence of a longitudinal change in cortical atrophy might be due to the short follow-up duration that is not enough for pathological processes to cause a measurable effect. MRI techniques sensitive to more subtle microstructural changes at the level of the neuronal processes can provide earlier markers preceding neuronal loss. ${ }^{23}$

Despite our small sample size $(n=13)$ at baseline and at follow-up $(n=12)$, we were able to observe changes in the anterior and posterior $\mathrm{CC}$ in as early as 7 months. It is likely that the $\mathrm{CC}$ plays an important role as the disease progresses during the manifest stages and calls for further examination of this region as a biomarker against which potential therapeutic agents can be evaluated. Movement during MRI acquisition was excessive in 2 patients, which led us to exclude them. Cognitive data were also not collected for the HC and no follow-up scanning was done, which prevented us from comparing the rate of GM and WM changes between groups. A larger sample size is required to establish the usefulness of short-term follow-up to assess changes in gray and WM areas as part of the neurodegenerative process in $\mathrm{HD}$, but the data from this small sample suggest it will be practical to use these changes as a measure of disease progression.

\section{References}

1. MacDonald ME, Ambrose CM, Duyao MP, et al. A novel gene containing a trinucleotide repeat that is expanded and unstable on Huntington's disease chromosomes. Cell 1993;72:971-83.

2. Hersch S, Rosas HD, Ferrante RJ. Neuropathology and Pathophysiology of Huntington's Disease. New York: McGraw-Hill; 2004.

3. Rosas HD, Salat DH, Lee SY, et al. Cerebral cortex and the clinical expression of Huntington's disease: complexity and heterogeneity. Brain 2008;131:1057-68.

4. Tabrizi SJ, Scahill RI, Owen G, et al. Predictors of phenotypic progression and disease onset in premanifest and early-stage Huntington's disease in the TRACK-HD study: analysis of 36-month observational data. Lancet Neurol 2013;12:637-49.

5. Stout JC, Paulsen JS, Queller S, et al. Neurocognitive signs in prodromal Huntington disease. Neuropsychology 2011;25:1-14.

6. McColgan P, Seunarine K, Gregory S, et al. Topological length of white matter connections predicts their rate of atrophy in premanifest Huntington's disease. JCI Insight 2017;2:92641.
7. Basser PJ, Mattiello J, LeBihan D. Estimation of the effective self-diffusion tensor from the NMR spin echo. J Magn Reson B 1994;103:247-54.

8. Poudel GR, Stout JC, Domínguez D JF, et al. Longitudinal change in white matter microstructure in Huntington's disease: The IMAGE-HD study. Neurobiol Dis 2015;74: 406-12.

9. Unified Huntington's Disease Rating Scale: Reliability and consistency. Huntington study group. Mov Disord 1996;11:136-42.

10. Tabrizi SJ, Langbehn DR, Leavitt BR, et al. Biological and clinical manifestations of Huntington's disease in the longitudinal TRACK HD study: cross-sectional analysis of baseline data. Lancet Neurol 2009;8:791-801

11. Nasreddine ZS, Phillips NA, Bédirian V, et al. The Montreal cognitive assessment, MoCA: a brief screening tool for mild cognitive impairment. J Am Geriatr Soc 2005;53:695-9.

12. Smith A. The Symbol Digit Modalities Test (SDMT): Manual. Los Angeles, CA: WPS; 1973.

13. Smith SM, Jenkinson M, Johansen-Berg H, et al. Tract-based spatial statistics: voxelwise analysis of multi-subject diffusion data. Neuroimage 2006;31:1487-505.

14. Nichols TE, Holmes AP. Nonparametric permutation tests for functional neuroimaging: a primer with examples. Hum Brain Mapp 2002; 15:1-25.

15. Madhyastha T, Mérillat S, Hirsiger S, et al. Longitudinal reliability of tract-based spatial statistics in diffusion tensor imaging. Hum. Brain Mapp. 2014;35:4544-55.

16. Fischl B. FreeSurfer. Neuroimage 2012;62:774-81.

17. Muhlau M, Weindl A, Wolschlager AM, et al. Voxel-based morphometry indicates relative preservation of the limbic prefrontal cortex in early Huntington disease. J Neural Transm 2007;114:36772 .

18. R. DellaNave A., Ginestroni C., Tessa M., et al. Regional distribution and clinical correlates of white matter structural damage in Huntington disease: a tract-based spatial statistics study. Am J Neuroradiol 2012;31:1675-81.

19. Bourbon-Teles J, Bells S, Jones DK, et al. Myelin breakdown in human Huntington's disease: multi-modal evidence from diffusion MRI and quantitative magnetization transfer. Neuroscience 2019;403:79-92.

20. Mazerolle E, D’Arcy RC, Beyea SD. Detecting functional magnetic resonance imaging activation in white matter: interhemispheric transfer across the corpus callosum. BMC Neurosci 2008; 9:84.

21. Turken U, Whitfield-Gabrieli S, Bammer R, et al. Cognitive processing speed and the structure of white matter pathways: convergent evidence from normal variation and lesion studies. Neuroimage 2008;42:1032-44.

22. Tabrizi SJ, Reilmann R, Roos RA, et al. Potential endpoints for clinical trials in premanifest and early Huntington's disease in the TRACK-HD study: analysis of 24 month observational data. Lancet Neurol 2012;11:42-53.

23. Zhang H, Schneider T, Wheeler-Kingshott CA, et al. NODDI: practical in vivo neurite orientation dispersion and density imaging of the human brain. Neuroimage 2012;61:1000-16.

24. Leemans A, Jones DK. The B-matrix must be rotated when correcting for subject motion in DTI data. Magn Reson Med 2009;61(6):1336-1349. 